\title{
Meningioma do nervo óptico simulando progiressão de dano axonal glaucomatoso: relato de caso
}

\author{
Optic nerve meningioma mimicking progression of glaucomatous \\ axonal damage: A case report
}

\author{
KenzoHokazono ${ }^{1}$ \\ Frederico Castelo Moura ${ }^{2}$ \\ Mário Luiz Ribeiro Monteiro ${ }^{3}$
}

Trabalho realizado na Divisão de Clínica Oftalmológica do Hospital das Clínicas da Faculdade de Medicina da (USP) - São Paulo (SP) - Brasil.

Médico Preceptor da Divisão de Clínica Oftalmológica das Clínicas Faculdade de Medicina da Universidade de São Paulo - USP - São Paulo (SP) - Brasil.

${ }^{2}$ Doutor em Oftalmologia do Setor de Neuroftalmologia e Órbita pela Faculdade de Medicina da USP - São Paulo (SP) - Brasil.

Professor Livre Docente, Chefe dos Serviços de Neuroftalmologia e Doenças da Órbita pela Faculdade de Medicina da USP - São Paulo (SP) - Brasil.

Endereço para correspondência: Frederico Castelo Moura. Rua Arruda Alvim, 297/152 - São Paulo (SP) CEP 05410-020

E-mail: fredcastelo@terra.com.br

Recebido para publicação em 21.02.2008

Última versão recebida em 21.04.2008

Aprovação em 07.05.2008

\begin{tabular}{|l|}
\hline RESUMO \\
\hline O objetivo é relatar um caso de meningioma da bainha do nervo óptico \\
localizado na região do canal óptico simulando progressão de dano axonal \\
glaucomatoso. Paciente de 60 anos em tratamento para glaucoma primário \\
de ângulo aberto apresentou perda progressiva do campo visual e aumento \\
da escavação do disco óptico à esquerda. Devido à rapidez e à assimetria da \\
progressão do dano axonal, por causa da redução da acuidade visual e do \\
aparente bom controle pressórico uma lesão compressiva do nervo óptico foi \\
suspeitada. A tomografia computadorizada de órbitas foi normal, entretanto \\
a imagem por ressonância magnética evidenciou um meningioma da bainha \\
do nervo óptico na região do canal óptico. Afecções compressivas da via \\
óptica anterior são causas incomuns de escavação do disco óptico, entretanto \\
podem simular uma neuropatia glaucomatosa e devem ser lembradas nos \\
pacientes com suspeita de glaucoma de baixa pressão intra-ocular ou em \\
tratamento para glaucoma que apresentem evolução atípica da perda visual. \\
\hline
\end{tabular}

Descritores: Meningioma; Neoplasia/patologia; Nervo óptico; Glaucoma; Tomografia computadorizada por raios X; Órbita; Acuidade visual; Humano; Feminino; Adulto; Relatos de casos [Tipo de publicação]

\section{INTRODUÇÃO}

O glaucoma primário de ângulo aberto é uma neuropatia óptica progressiva caracterizada por perda da camada de fibras nervosas da retina (CFNR), aumento da escavação do nervo óptico e defeito de campo visual (CV) correspondente ${ }^{(1)}$. A pressão intra-ocular (PIO) é o principal fator etiopatogênico no glaucoma e quanto maiores os seus valores, maior a prevalência e o risco de progressão da doença ${ }^{(2)}$. Embora a PIO seja o parâmetro clínico mais facilmente analisado, a suspeita de progressão da doença é baseada particularmente no aumento da escavação do nervo óptico e na progressão do defeito de CV.

O objetivo deste trabalho é relatar uma paciente com diagnóstico prévio de glaucoma primário de ângulo aberto que apresentou progressão do defeito perimétrico e aumento importante da escavação do nervo óptico de um dos olhos decorrentes de um meningioma da bainha do nervo óptico localizado no canal óptico. Revisamos também as principais características clínicas das neuropatias ópticas compressivas que permitem diferenciá-las do glaucoma.

\section{RELATO DO CASO}

Paciente de 62 anos, sexo feminino, brasileira, branca, natural e procedente de São Paulo, dona de casa, foi encaminhada para o setor de Neuroftal- 
mologia do Hospital das Clínicas da Faculdade de Medicina da Universidade de São Paulo para investigação de perda progressiva da visão do olho esquerdo (OE). A paciente vinha fazendo tratamento no ambulatório de Glaucoma da mesma instituição há um ano. $\mathrm{Na}$ avaliação inicial naquele serviço apresentava acuidade visual (AV) de 20/20 em ambos os olhos, ausência de alterações biomicroscópicas do segmento anterior, ângulo do seio camerular aberto nos $360^{\circ}$ nos dois olhos à gonioscopia e curva tensional diária apresentando limites pressóricos máximos de $21 \mathrm{mmHg}$ no olho direito (OD) e $22 \mathrm{mmHg}$ no OE. A espessura corneana média foi 531 (525-541) $\mu \mathrm{m}$ no OD e 535 (531-539) $\mu \mathrm{m}$ no OE. A perimetria computadorizada (Humphrey, programa 24-2 SITA Standard threshold test) revelou no OD um defeito superior esboçando um escotoma arqueado e um degrau nasal, e no OE uma depressão difusa da sensibilidade acometendo principalmente o hemicampo inferior (Figura 1A). Relato do exame de fundo de olho daquela época indicava a presença de escavação com diâmetros vertical e horizontal de $0,6 \times 0,6$ em ambos os olhos. Nessa ocasião, foi feito o diagnóstico de glaucoma primário de ângulo aberto e iniciado o tratamento com colírio brimatoprost uma vez ao dia em ambos os olhos.

Nos meses seguintes, a despeito do controle pressórico, apresentou perda visual e progressão do defeito perimétrico à esquerda (Figura 1B). Quando avaliada no setor de Neuroftalmologia, referiu que a perda visual havia sido lenta e progressiva no $\mathrm{OE}$, sem dor ocular ou proptose associada. Ao exame apresentava AV corrigida de 20/20 no OD e de projeção luminosa no OE. Não havia alterações ao exame ocular externo, à biomiocroscopia do segmento anterior, ao exame da motilidade ocular extrínseca ou à exoftalmometria. As pupilas eram isocóricas e fotorreagentes, mas havia um defeito pupilar aferente relativo importante à esquerda. A PIO media $12 \mathrm{mmHg}$ no $\mathrm{OD}$ e $13 \mathrm{mmHg}$ no OE. À fundoscopia, o disco óptico do OD apresentava escavação de $0,6 \times 0,6$ e do $\mathrm{OE}$ escavação de $0,9 \times 0,9$, associada à palidez difusa da rima neural (Figura 1C).

Devido ao aumento da escavação, à piora acentuada do defeito do $\mathrm{CV}$, à perda de $\mathrm{AV}$ e à assimetria acentuada dos achados, a despeito do controle adequado da pressão ocular, foi considerado o diagnóstico de uma lesão compressiva do nervo óptico esquerdo e solicitada tomografia computadorizada (TC) de órbitas (Figura 2A) que se mostrou normal. A análise da TC realizada revelou, no entanto, que as regiões do nervo óptico intracraniano e do canal óptico não haviam sido estudadas adequadamente. Em decorrência disto, foi solicitada imagem por ressonância magnética (IRM) de alta resolução para estudar aquelas regiões. $\mathrm{O}$ exame revelou uma massa hipo-intensa em cortes enfatizando T1 com supressão de gordura e hiperintensa após injeção de agente paramagnético (gadolíneo), compatível com o diagnóstico de meningioma da bainha do nervo óptico acometendo a região do canal óptico e nervo óptico intracraniano (Figura 2B).

\section{DISCUSS ÃO}

A neuropatia óptica glaucomatosa é a causa mais comum do aumento da relação escavação/diâmetro de disco (E/D), sendo este um dos elementos semiológicos mais importantes para o seu diagnóstico. Entretanto, algumas neuropatias ópticas não glaucomatosas podem ocasionalmente levar a um aumento da relação $\mathrm{E} / \mathrm{D}$, mesmo com pressão intra-ocular dentro dos limites normais ${ }^{(3)}$ incluindo: a neuropatia óptica isquêmica anterior forma arterítica ${ }^{(4)}$, a neuropatia por aumento fusiforme da artéria carótida intracraniana, neuropatias ópticas hereditárias ${ }^{(5)} \mathrm{e}$ lesões compressivas da via óptica anterior ${ }^{(6)}$. Todas estas condições podem levar à confusão diagnóstica com glaucoma, dependendo da experiência do examinador ${ }^{(1)}$.

Das neuropatias ópticas não glaucomatosas que podem se associar com escavação, as mais importantes são as neuropatias compressivas. Uma vez que sua evolução é usualmente lenta e progressiva e como ocorrem geralmente em pacientes idosos, a possibilidade de confusão com glaucoma é muito mais importante do que em outras neuropatias ópticas. A associação entre escavação do nervo óptico e lesões compressivas da via óptica anterior foi previamente descrita por diversos autores. Alguns autores apresentaram uma série de 16 pacientes com lesão compressiva da via óptica anterior que desenvolveram escavação não glaucomatosa do nervo óptico ${ }^{(7)}$. Entre os achados semiológicos relevantes, chamaram à atenção a perda visual desproporcional à escavação e a relação E/D maior que 0,49 encontradas nestes pacientes. Através de análise quantitativa da escavação do nervo óptico realizada por meio de estereofotografias, alguns autores demonstraram objetivamente que lesões compressivas da via óptica anterior podem causar aumento da escavação do nervo óptico na ausência de pressão intra-ocular elevada ${ }^{(8)}$. Esses autores demonstraram uma diferença média de 0,13 na relação E/D entre os olhos dos pacientes com lesão unilateral do nervo óptico e de 0,04 dos indivíduos controles $(\mathrm{p}=0,0001)$. A assimetria da relação $\mathrm{E} / \mathrm{D}$ entre os dois olhos de um paciente com compressão de nervo óptico unilateral é um achado convincente de que aumento da escavação é uma característica adquirida.

Apesar da diferenciação entre escavação não glaucomatosa e glaucomatosa usualmente ser difícil, alguns fatores podem ajudar o diagnóstico. Assim, pacientes jovens, redução importante da $\mathrm{AV}$, defeitos de $\mathrm{CV}$ respeitando a linha média vertical e palidez da rima neurorretiniana devem levar à suspeita de compressão da via óptica anterior, mesmo quando existe escavação do disco óptico ${ }^{(9)}$. Outros autores através de análise estereofotográficas do disco óptico investigaram as características que ajudariam a distinguir entre escavação glaucomatosa e não glaucomatosa ${ }^{(3)}$. Os resultados sugeriram que a palidez da rima neurorretiniana é um sinal altamente específico (94\%) para neuropatia óptica não glaucomatosa e que o afilamento completo (focal ou difuso) da rima neurorretiniana é específico para o glaucoma. Por outro lado, o afilamento parcial da rima neurorretiniana e a exposição da lâmina 

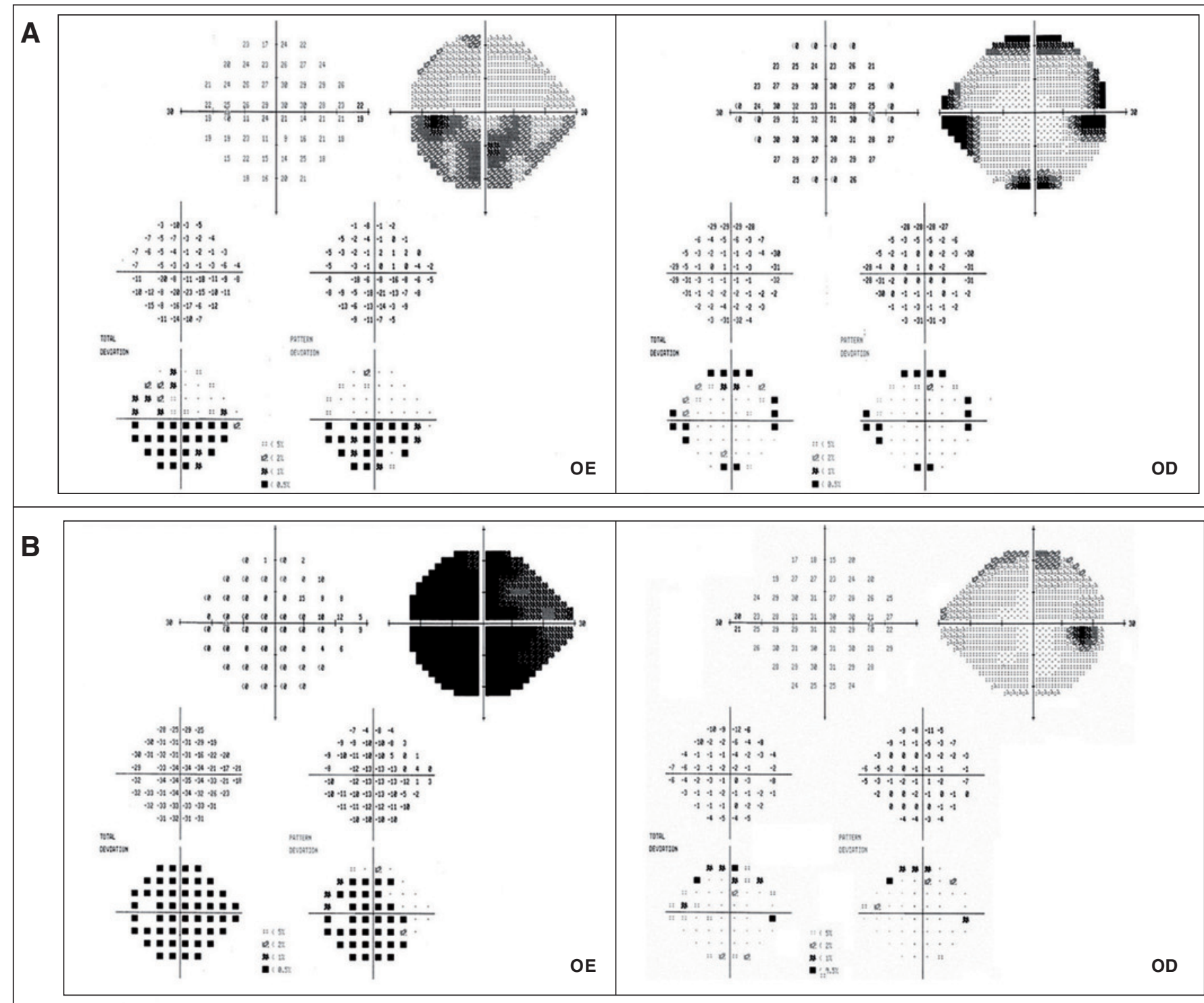

\section{C}

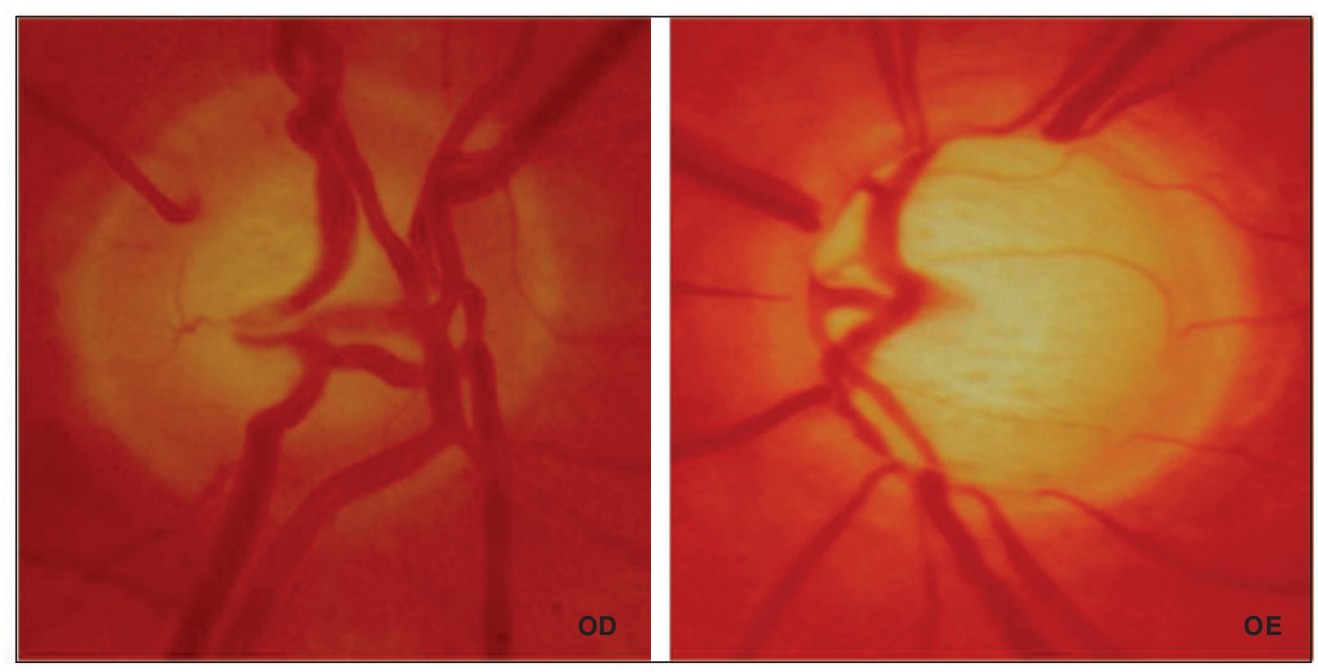

Figura 1 - A) Campo visual computadorizado inicial evidenciando defeito em ambos os olhos, mais evidente à esquerda. B) Campo visual 1,5 ano após o início do tratamento para glaucoma evidenciando piora acentuada no olho esquerdo. C) Fotografia dos discos ópticos mostrando escavação grande em ambos os olhos com assimetria acentuada de escavação (maior do que 0,3 ). 


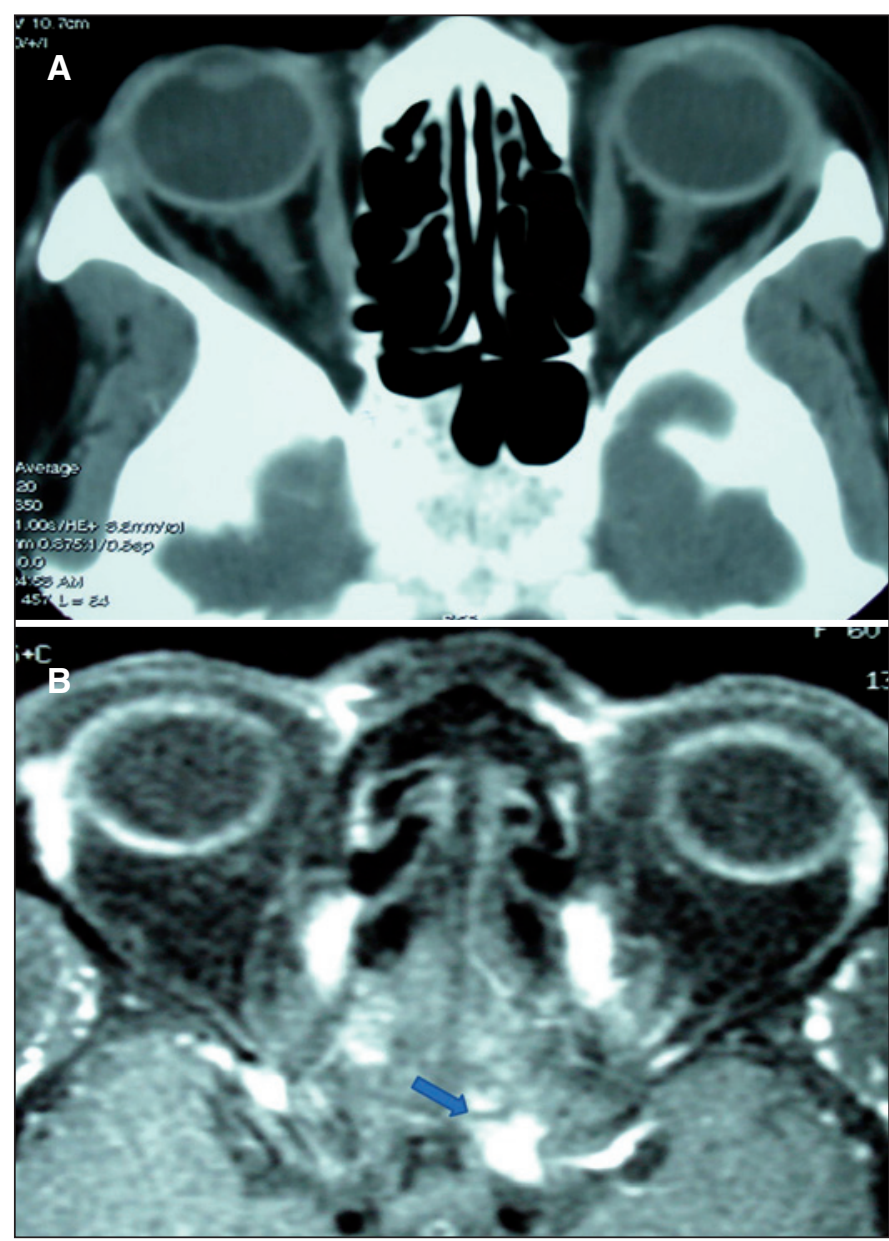

Figura 2 - A) tomografia computadorizada das órbitas sem evidenciar alterações. B) imagem por ressonância magnética após a injeção de gadolíneo mostrando processo expansivo com sinal aumentado na região do canal óptico e nervo óptico intracraniano compatível com meningioma (seta).

cribiforme não foram úteis em separar os dois tipos de escavação patológica ${ }^{(3)}$. Em recente publicação, autores encontraram uma prevalência de lesões compressivas da via óptica significativamente maior nos pacientes com diagnóstico clínico de glaucoma de pressão normal do que nos com glaucoma primário de ângulo aberto (respectivamente, $6,5 \%$ e $0 \%$; $\mathrm{p}=0,039)^{(10)}$. Os autores sugeriram que a investigação neuroradiológica é a medida mais eficaz nos pacientes com glaucoma de pressão normal, principalmente na ausência dos sinais clássicos das afecções neuroftálmicas como palidez do disco óptico e defeito de $\mathrm{CV}$ respeitando o meridiano vertical.

Além dos sinais já citados, escavação não glaucomatosa do nervo óptico também pode se associar aos achados clínicos não característicos do glaucoma como, por exemplo, progressão muito rápida da perda de $\mathrm{AV}$ e de $\mathrm{CV}$ estando a PIO normal, discromatopsia e defeito pupilar aferente relativo. A associação desses achados neuroftalmológicos e das características clínicas do nervo óptico podem conduzir a correta investigação por meio de exames de neuro-imagem de tal forma a diagnosticar precocemente a causa da escavação patológica do nervo óptico e orientar o tratamento adequado.

\section{ABSTRACT}

To report a case of an optic nerve sheath meningioma located at the optic canal area mimicking the progression of a glaucomatous axonal damage. A 60-year-old female patient developed progressive visual field loss and enlargement of the optic disc cup in the left eye while in treatment for primary openangle glaucoma. Because of the rapid progression of axonal loss, the asymmetry of the visual field defect and the fact that intraocular pressure had been under control, a compressive optic neuropathy was suspected. A computerized tomography scan of the orbit was normal but a high-resolution magnetic resonance imaging demonstrated an optic nerve sheath meningioma located at the optic canal area. Compressive lesions of the anterior visual pathways are uncommon causes of optic disc cupping but should be considered in patients suspected of or undergoing treatment for glaucoma that present with atypical progression of visual loss.

Keywords: Meningioma; Neoplasms/pathology; Optic nerve; Glaucoma; Tomography, x-ray computed; Orbit; Visual acuity; Human; Female; Adult; Case reports [Publication type]

\section{REFERÊNCIAS}

1. Weinreb RN, Khaw PT. Primary open-angle glaucoma. Lancet 2004;363 (9422):1711-20. Comment in: Lancet. 2004;364(9442):1311-2.

2. Comparison of glaucomatous progression between untreated patients with normal-tension glaucoma and patients with therapeutically reduced intraocular pressures. Collaborative Normal-Tension Glaucoma Study Group. Am J Ophthalmol 1998;126(4):487-97. Erratum in: Am J Ophthalmol. 1999;127(1): 120. Comment in: Am J Ophthalmol. 1998;126(4):578-81; Am J Ophthalmol. 1999;128(6):776-7; Am J Ophthalmol. 1999;127(5):623-5; Am J Ophthalmol. 1999;127(5):625-6.

3. Trobe JD, Glaser JS, Cassady J, Herschler J, Anderson DR. Nonglaucomatous excavation of the optic disc. Arch Ophthalmol. 1980;98(6):1046-50.

4. Quigley H, Anderson DR. Cupping of the optic disc in ischemic optic neuropathy. Trans Sect Ophthalmol Am Acad Ophthalmol Otolaryngol. 1977; 83(5):755-62.

5. Ortiz RG, Newman NJ, Manoukian SV, Diesenhouse MC, Lott MT, Wallace DC. Optic disk cupping and electrocardiographic abnormalities in an American pedigree with Leber's hereditary optic neuropathy. Am J Ophthalmol. 1992;113(5):561-6.

6. Kalenak JW, Kosmorsky GS, Hassenbusch SJ. Compression of the intracranial optic nerve mimicking unilateral normal-pressure glaucoma. J Clin Neuroophthalmol 1992;12(4):230-5; discussion 236-7.

7. Kupersmith MJ, Krohn D. Cupping of the optic disc with compressive lesions of the anterior visual pathway. Ann Ophthalmol. 1984;16(10):948-53.

8. Bianchi-Marzoli S, Rizzo JF $3^{\text {rd }}$, Brancato R, Lessell S. Quantitative analysis of optic disc cupping in compressive optic neuropathy. Ophthalmology. 1995;102(3):436-40. Comment in: Ophthalmology. 1995;102(11):1577-8.

9. Greenfield DS, Siatkowski RM, Glaser JS, Schatz NJ, Parrish RK $2^{\text {nd }}$. The cupped disc. Who needs neuroimaging? Ophthalmology. 1998;105(10):186674. Comment in: Ophthalmology. 1999;106(5):855.

10. Ahmed, II, Feldman F, Kucharczyk W, Trope GE. Neuroradiologic screening in normal-pressure glaucoma: study results and literature review. J Glaucoma. 2002;11(4):279-86. Comment in: J Glaucoma. 2002;11(4):277-8. 